\section{Turning on cholera RTX}

Vibrio cholerae secretes several toxins, including the actin-disrupting toxin RTX. Similarly to other toxins, RTX undergoes autoproteolysis once it enters the host cell, releasing an effector domain. The cysteine-protease domain (CPD) of RTX is thought to mediate autoproteolysis in response to the eukaryote-specific small molecule inositol hexakisphosphate $\left(\operatorname{InsP}_{6}\right)$,

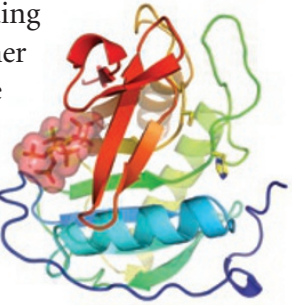
but the exact mechanism remained a mystery.

Garcia, Bogyo and colleagues have now solved the crystal structure of the RTX CPD in complex with $\mathrm{InsP}_{6}$, revealing a clever mechanism for RTX autoactivation. Ins $\mathrm{P}_{6}$ binds to a conserved basic cleft away from the protease active site, indicating that it is not a cofactor for catalysis. In addition, kinetic analysis confirmed that $\mathrm{Ins}_{6}$ binding is independent of substrate binding. A series of mutations in the region between the active site and $\mathrm{Ins}_{6}$ binding site indicated that $\mathrm{Ins}_{6} \mathrm{P}_{6}$ binding induces an allosteric switch that leads to autoprocessing and release of the effector domain. It had been previously suggested that $\operatorname{Ins}_{6}$ binding controls exposure of the active site, and the authors here demonstrate that productive binding of $\mathrm{InsP}_{6}$ is indeed needed to expose the protease active site cysteine to substrates and inhibitors, indicating that the allosteric switch provides a way of protecting the active site sulfhydryl group until the toxin enters the cell. As $\mathrm{InsP}_{6}$ is found only in eukaryotes, this mechanism is likely to be used by other bacterial pathogens to ensure that their effectors are activated only when inside the host cell. (Science 322, 265-267, 2008)

$\mathrm{MH}$

\section{Proteins caught on the hop}

The ability of sequence-specific DNA binding proteins to locate their target in a vast excess of nonspecific DNA is remarkable. Much theoretical effort and biochemical study have led to a model in which a protein binds initially to any DNA sequence and then slides along the DNA in a one-dimensional search. If no specific sequence is encountered, it can either hop along the same DNA segment or jump to a nearby segment, before resuming sliding. Longer dissociation events allow an even greater volume to be tested, but 'knowledge' about what space has already been explored is lost. In a recent paper by van den Broek et al., a single-molecule approach is used to examine how DNA conformation can influence the search process. The authors stretched a linearized plasmid with a single EcoRV site between two optical tweezers, which were manipulated to yield different degrees of DNA coiling (either extended, in a relaxed random configuration or tightly squeezed) and determined the association rates with EcoRV endonuclease. The association rate was highest when the DNA was in its maximal free, random state. As the DNA is extended the rate is impeded by the lower frequency of intersegmental jumps, whereas in the squeezed configuration the local density of DNA segments is reduced relative to the random state, again impacting the rate. The data support a model in which coiling helps to accelerate changes in the neighboring DNA configuration, such that they occur much faster than the time needed for a protein to escape a segment. Consequently, intersegmental jump events are important and reduce repeated binding of the same segment of DNA. In addition, the authors propose that the closed conformation adopted by unbound EcoRV could facilitate uniform spreading of the enzyme throughout the cell. (Proc. Natl. Acad. Sci. USA 105, 15738-15742, 2008)

AKE

Written by Angela K. Eggleston, Joshua M. Finkelstein, Maria Hodges \& Sabbi Lall

\section{Sweet acetylation}

During fasting, liver glucose production is regulated through a number of factors and is first upregulated then eventually downregulated as ketogenesis is initiated. Montminy and colleagues have now explored how the key transcription factors CREB-regulated transcription coactivator 2 (CRTC2) and forkhead box O1 (FOXO1) are able to give such complex regulatory output. Using specific reporters, the authors showed that CRTC2 and FOXO1 activities increase in a sequential fashion: CRTC2 activity is high during early fasting, with FOXO1 activity predominating at later times. Whereas CRTC2 activity mirrors its protein levels, FOXO1 levels remain constant during fasting. In the former case, both fasting and glucagon treatment triggered CRTC2 acetylation and thus stabilization. This acetylation is primarily p300 mediated and occurs at a site previously known to be ubiquitinated. Indeed, fasting and glucagon treatment increased the CRTC2-p300 interaction, with p300 association with CRTC2 being dependent on decreasing p300 phosphorylation by the Ser/Thr kinase SNF1-like kinase 2 (SIK2). Manipulating these interactions and modifications could affect both reporter-gene expression and glucose output in hepatocytes. So what reverses CRTC2 acetylation to decrease glucose production? The authors investigated the possibility that the deacetylase sirtuin 1 (SIRT1) might be involved and found that SIRT1 expression or activation by SRT1720 or resveratrol reduced CRTC2 acetylation and CRTC2-reporter activity and decreased blood glucose levels. These activities were dependent on the presence of wild-type CRTC2, but not a protein mutated at the above-mentioned acetylation site. In sum, the study illustrates and elucidates how posttranslational modifications can affect the intricate alterations that must occur in response to changing nutritional needs. (Nature advance online publication 5 October 2008, doi:10.1038/nature07349)

$S L$

\section{Just a trim}

Histone proteins interact with DNA to form nucleosomes, the key component of chromatin. Nucleosome core particles contain two copies of each of the four core histones, and two of these- $\mathrm{H} 3$ and $\mathrm{H} 4$ - have long 'tails' that extend out of the core. The amino acid side chains in these histone tails can be covalently modified, and the location and density of these modifications change during the differentiation of embryonic stem cells (ESCs). In a recent paper, Duncan et al. identified a truncated version of histone $\mathrm{H} 3$ that appeared several days after treating mouse ESCs with retinoic acid. Further experiments revealed that the $\mathrm{N}$ terminus of $\mathrm{H} 3$ was being proteolytically cleaved by an endopeptidase. The authors identified the source of the H3-protease activity in fractionated nuclear extracts derived from differentiating mouse ESCs as cathepsin L, a cysteine protease. Low concentrations of a cysteine-protease inhibitor, but not a serine-protease inhibitor, inhibited histone H3-cleavage activity in vitro; in addition, RNAi knockdown or use of a cell-permeable specific inhibitor of cathepsin $L$ reduced histone $\mathrm{H} 3$ cleavage in differentiating ESCs. The authors also showed that recombinant cathepsin L, but not cathepsin B or $\mathrm{K}$, could reproduce the cleavage pattern seen in the cellular experiments, lending further credence to the hypothesis that this protease was responsible for the histone $\mathrm{H} 3$ cleavage during ESC differentiation. Whether other histones are selectively cleaved during development or differentiation, and whether other histone-modifying proteins can regulate this process remain to be determined. (Cel/ 135, 284-294, 2008) $J M F$ 